\title{
Mathematical model for kinetics of alkali-silica reaction in concrete
}

\author{
Zdeněk P. Bažant ${ }^{\mathrm{a}, *}$, Alexander Steffens ${ }^{\mathrm{b}}$ \\ ${ }^{a}$ Department of Civil Engineering and Materials Science, Northwestern University, Evanston, IL 60208-3109, USA \\ ${ }^{\mathrm{b}}$ Northwestern University, Evanston, IL 60208-3109, USA
}

Received 29 August 1999; accepted 17 December 1999

\begin{abstract}
Vast though the literature on the chemistry of the alkali-silica reaction (ASR) in concrete has become, a comprehensive mathematical model allowing quantitative predictions seems lacking. The present study attempts a step toward this goal. While two distinct problems must be dealt with, namely, (1) the kinetics of the chemical reaction with the associated diffusion processes and (2) fracture mechanics of the damage process, only the former is addressed here. The analysis is focused on the recent attempts by C. Meyers and W. Jin to incorporate ground waste glass (mainly, bottle glass) into concrete. With minor adjustments, though, the model can be applied to ASR in natural aggregates as well. A characteristic unit cubic cell of concrete containing one spherical glass particle is analyzed. A spherical layer of basic ASR gel grows radially inward into the particle, controlled by diffusion of water toward the reaction front. Modification of the solution for the case of mineral aggregates with veins of silica is also indicated. Imbibition of additional water from the adjacent capillary pores, which causes swelling of the gel, is described as a second diffusion process, limited by the development of pressure due to resistance of concrete to expansion. The water used up to form the basic ASR gel and imbibed to cause its swelling appears as a sink term in the non-linear diffusion equation for the global water transport through a concrete structure. The differential equations are integrated numerically. The study of the effects of various parameters provides improved understanding of the ASR, and especially the effect of glass particle size. Full prediction will require measurements of some parameters of the reaction processes. (C) 2000 Elsevier Science Inc. All rights reserved.
\end{abstract}

Keywords: Concrete; Alkali-aggregate reaction; Glass; Diffusion; Durability; Expansion; Mechanical properties; Waste recycling; Reaction kinetics; Mathematical modeling

\section{Introduction}

The excessive expansion and disintegration of some concrete made of cements of a relatively high alkali content and of certain silica-containing aggregates can be explained by the so-called alkali-silica reaction (ASR) - a chemical reaction of the alkalis with the hydrous forms of the silica present in the mineral constituents, discovered in 1940 by Stanton [1] and described early by Blanks [2] and Meissner [3]. The ASR produces a soft viscous substance called the ASR gel, which has been observed to appear in pop-outs or to exude from cracks in concrete structures. The chemistry of ASR has been extensively researched in many laboratories during the last several decades, and much has been learned; see, e.g., Glasser [4], Glasser and Kataoka [5], Dron and Brivot [6-8], Dron et al. [9], Vivian [10],

\footnotetext{
* Corresponding author. Tel.: +1-847-491-4025; fax: +1-847-467-1078.

E-mail address: z-bazant@nwu.edu (Z.P. Bažant).
}

Swenson [11], Poole [12], Swamy [13,14], and Gartner and Jennings [15].

Unfortunately, the problem is very complex, influenced by many factors. This makes it impossible to make realistic predictions solely by intuitive reasoning based on the present qualitative knowledge of the ASR chemistry. The factors that are important under various circumstances, and those that are not, cannot be sorted out without a comprehensive mathematical model. Such a model seems to be unavailable at present. Its simplified formulation is taken as the objective of this study.

There are two basic problems for a comprehensive model: (1) the modeling of the kinetics of the chemical and diffusional processes involved, and (2) the modeling of the mechanical damage to concrete, which calls for fracture mechanics. Only the former problem is addressed in this study, aimed at the development of a comprehensive model. Various aspects of the former problem have already been studied, e.g., by Groves and Zhang [16], Prezzi et al. [17] and $\mathrm{Xi}$ et al. [18]. A comprehensive model for the latter problem 
has been outlined at two recent conferences $[19,20]$ and developed in more detail in Ref. [21]. Of course, an amalgamation of the models for both problems is planned.

The present investigation was stimulated by the recent attempts of Meyer and Baxter [22] and Jin [23] at the Columbia University to find a way of incorporating waste glass (mainly, bottle glass) into concrete (a glass content of about $10 \%$ of the volume of concrete is contemplated). Meyer et al. made a startling discovery - reducing the glass particle worsens the damage to concrete only down to a particle size of 1 to $2 \mathrm{~mm}$. If the particle size is reduced further, the trend is reversed, i.e., the damage diminishes. For particle sizes about $0.1 \mathrm{~mm}$, no damage is detected. An elementary explanation of the reversal of particle size effect in terms of fracture mechanics was proposed in Refs. $[19,20]$, and a more sophisticated fracture mechanics explanation was given in Ref. [21]. Xi et al.'s [18] mathematical analysis showed that some aspects of the reversal can also be attributed to diffusional phenomena.

The aforementioned findings have been obtained solely with the recently introduced accelerated ASTM test of 2week duration, through measurements of volume expansion and compression strength. It is not at all clear whether this startling reversal of particle size effect, which would render the incorporation of waste glass harmless, occurs also for the normal lifetimes of concrete structures. To decide this question, the kinetics of ASR and the diffusional processes involved must be modeled mathematically. This is the motivation of the present investigation.

\section{Review of the chemistry of ASR}

The main constituent of most aggregates is silicon dioxide, $\mathrm{SiO}_{2}$. It occurs as crystalline silica polymorphs mainly in the form of mineral quartz, but also in the form of tridymite or cristobalite. Quartz is basic and has chemically stable bonds, and thus it is relatively unreactive and unaffected by most strong acids or alkalis. Tridymite or cristobalite have more open crystalline frameworks that exhibit substantially enhanced reactivity towards alkali. However, $\mathrm{SiO}_{2}$ may also occur in disordered frameworks.

The thermodynamic metastability and the comparatively open, disordered structures of these poorly crystallized silicas also give rise to an enhanced potential for reaction with cement alkalis. Moreover, a unique structural relationship between $\mathrm{SiO}_{2}$ and $\mathrm{H}_{2} \mathrm{O}$ enables 'water' to substitute to some extent for silica, thus forming amorphous hydrous silica, which may be very reactive in the presence of alkalis. Opal, chert, chalcedony and glasses are examples of such poorly crystallized, hydrous silica. There is no reliable way of predicting how susceptible the diverse siliceous aggregates are to ASR.

The alkalis are in cement supplied in the form of sodium oxide, $\mathrm{Na}_{2} \mathrm{O}$, and potassium oxide, $\mathrm{K}_{2} \mathrm{O}$. These oxides, initially found diffused within the anhydrous phases of the cement, dissolve in the pore liquid during the process of hydration, as described by $\mathrm{Na}^{+}+\mathrm{OH}^{-}$and $\mathrm{K}^{+}+\mathrm{OH}^{-}$, forming sodium, potassium and hydroxyl ions, respectively. As these ions do not take part in the formation of normal cement hydration products, they accumulate in the pore solution.

The anhydrous calcium oxide of the cement, $\mathrm{CaO}$, reacts during hydration to yield calcium silicate hydrate, C-S-H, and calcium hydroxyl, $\mathrm{Ca}(\mathrm{OH})_{2}$. Whereas the C-S-H is not soluble, the $\mathrm{Ca}(\mathrm{OH})_{2}$ may dissolve in the pore liquid as $\mathrm{Ca}^{2+}+2 \mathrm{OH}^{-}$. Yet, the high alkali concentration due to $\mathrm{Na}^{+} \mathrm{OH}^{-}$and $\mathrm{K}^{+} \mathrm{OH}^{-}$renders calcium very insoluble. The $\mathrm{pH}$ of such pore fluids may well be greater than 12.4 , which is the approximate upper limit attainable by the solution of $\mathrm{Ca}(\mathrm{OH})_{2}$ at $20^{\circ} \mathrm{C}$.

Thus, the pore fluid of cement contains relatively high concentrations of hydroxyl ions, whose charge is mainly balanced by the aforementioned alkalis. It is the hydroxyl ion, and not the alkali ion, which initiates the chemical reaction of the silica.

The chemistry of this reaction is difficult to figure out in detail. The essential explanation of the chemical process was given by Powers and Steinour [24] and, in more detail, by Dent Glasser and Kataoka [5,25]. In their classical view, the reaction consists of topochemical interface processes. Recently, Dron and Brivot [6-8], and Dron et al. [9] argue that ASR involves mainly ionic reactions in the pore solution and speak of a 'transitory silica gel' formed of monomer species as a result of aggregate dissolution. Many different chemical equilibria are possible, depending on the $\mathrm{pH}$ of the pore solution and the ionic species present in it. However, it is far beyond the purpose of this study to discuss these complex reactions.

The reaction may be considered as a multi-stage process. It starts with the dissolution of silica on the surface of the aggregate particles. Each silicon atom is connected to the lattice by four siloxane bonds. Their rupture by $\mathrm{OH}^{-}$ions is of the topochemical type.

First, the oxygen atoms on the surface of the aggregate are hydroxylated, i.e, the siloxane bonds are broken and replaced by silanol bonds;

$\mathrm{Si}-\mathrm{O}-\mathrm{Si}+\mathrm{H}_{2} \mathrm{O} \rightarrow \mathrm{Si}-\mathrm{OH} \cdots \mathrm{OH}-\mathrm{Si}$.

Normally, the surface oxygen of siliceous aggregates are already hydroxylated up to a depth of several atoms or even tens of atoms. If these aggregates come into contact with the high-alkaline pore solution, their potential to undergo further hydroxylation is enhanced. This holds also for quartz but due to the strong bonds of its crystalline framework, the rate of hydroxylation is too slow for being considered on the normal engineering time scale. Yet the aforementioned poorly crystallized silica react much more rapidly.

Hydrous silica aggregates, as already mentioned, may of course already contain substantial silanol bonding. These silanol groups react readily with further hydroxyls;

$\mathrm{Si}-\mathrm{OH}+\mathrm{OH}^{-} \rightarrow \mathrm{SiO}^{-}+\mathrm{H}_{2} \mathrm{O}$. 
As more siloxane bonds are attacked, a gel-like layer forms on the surface of the aggregate. Some silica may even pass into the solution.

For highly alkaline solutions, the principal solute species is the monomer $\mathrm{H}_{2} \mathrm{SiO}_{4}{ }^{2-}$. Dron et al. [9] reproduced the dissolution of silica in experimental studies using the conditions of the ASTM C 289-71 test. Their results show that as the $\mathrm{pH}$ tends towards 11.2 , the monomer $\mathrm{H}_{3} \mathrm{SiO}_{4}{ }^{-}$ becomes exclusively a solute species.

The negatively charged species of this gel attract positive charges, which are present in the pore solution in the form of mobile species such as sodium, potassium, and calcium. They diffuse into the gel in sufficient numbers to balance the negatively charged groups.

The presence of these ions determines important properties of the gel. If the gel is formed in an environment rich in $\mathrm{Ca}^{2+}$, mainly this species will be taken up by the gel, soon forming particles of C-S-H as a separate constituent. Thus, the gel is transformed into a rigid and unreactive structure. This process may be considered similar to the pozzolanic reaction in concrete.

If the pore solution, however, is low in $\mathrm{Ca}^{2+}$, the gel takes up mainly $\mathrm{Na}^{+}$and $\mathrm{K}^{+}$. This results in a more viscous consistence of the gel. This gel will also imbibe further water, which will cause its large expansion.

If the gel is in contact with $\mathrm{CO}_{2}$, which occurs when it is exposed to the atmosphere, it carbonates. This is observed on the surface of cut concrete specimens that have undergone ASR. The ability to imbibe water and ionic species as $\mathrm{Na}^{+}$and $\mathrm{K}^{+}$cause the water contents, densities, and other physical properties to vary over wide ranges. It is very difficult to predict the species of gel that may form in a certain concrete.

\section{Formation of basic ASR gel from spherical glass particles}

To set up a model of ASR, it is inevitable to simplify the complex chemical reactions and diffusion processes involved. The role of water is fundamental for three reasons:

1. The pore water acts as the necessary transport medium for the mass transport of hydroxyl and alkali ions required by the reaction.

2. The expansion of the gel is essentially governed by the imbibition of water.

3. For the reaction to continue, water must be supplied by macro-diffusion through the pores of concrete.

For the sake of simplicity, the present simplified model will not specifically describe the individual behaviors of the sodium, potassium, and calcium ions. The solution in the pores will be assumed to have a low calcium ion content, which is necessary for the formation of the kind of hydrous gel that has the tendency to swell.
Aggregate particles in concrete may contain reactive silica in flaws, inclusions, and veins. If waste glass (mainly, bottle glass) is used as an aggregate [19-23], the whole particle is reactive. The shape and size of the particles may vary widely. The dissolution of the silica is of the topochemical type, that is, its progress depends on the geometrical properties of the aggregate, especially its reactive surface. In the following, equal-sized spherical particles of silica are considered. This is a reasonable simplification for waste glass in concrete but might be rather crude for natural aggregates. For roughly spherical particles, it may be assumed that the dissolution of silica progresses roughly in a uniform manner in the radial direction inward from the surface. The particles are assumed to be completely reactive, which holds true for waste glass. The reactive aggregate is assumed to be statistically distributed uniformly in the concrete matrix.

For mineral particles of highly irregular shape, the advance of ASR within the particle is rather non-uniform, depending on the surface shape and distribution of silica within the particle. This leads to the formation of flaws within the particle, filled by ASR gel. Expansion of the gel in such flaws is capable of cracking the mineral particle.

Eqs. (1) and (2) confirm that water, both in its molecular and ionized forms, is the driving force behind the dissolution of silica. This is the salient fact motivating the present modeling approach. Yet a stoichiometric relationship for water is very difficult to ascertain, due to the aforementioned great variety of chemical equilibria which are possible for different values of $\mathrm{pH}$.

If, as a simplification, the monomer $\mathrm{H}_{2} \mathrm{SiO}_{4}{ }^{-}$is considered to be the unique form of basic gel produced by the dissolution process, an approximate stoichiometric relationship between the reactants silicon dioxide, water in the form of hydroxyl ions, and the basic gel as reaction product, can nevertheless be figured out from the simplified chemical relation

$\mathrm{SiO}_{2}+2\left(\mathrm{OH}^{-}\right) \rightarrow \mathrm{H}_{2} \mathrm{SiO}_{4}^{2-}$.

As a crude but useful approximation, it can thus be stated that two water molecules are necessary to dissolve one silica atom. In the following, the resulting monomer $\mathrm{H}_{2} \mathrm{SiO}_{4}{ }^{-}$will be referred to as the 'basic' form of ASR gel.

The thermodynamics and kinetics of this reaction were studied by Dron and Brivot [6,7]. The reaction rate of the dissolution is very high compared to the rate of ASR observed in real concrete structures. This leads to the conclusion that the reaction rate is not governed by the difference in the chemical potentials but by another mechanism.

With the progress of dissolution, the size of the remaining unreacted silica particle decreases while a spherical layer (shell) of the reaction product, the ASR gel, grows around the particle. It is this layer through which further water molecules must diffuse in order to reach the reacting surface of the particle and dissolve more silica. This slows down the 
reaction tremendously and becomes the process governing the rate of dissolution. Thus, the rate of the dissolution can be determined by solving the diffusion problem.

The interaction of the reacting glass particle with the concrete matrix could be simplified in various ways. One is the so-called self-consistent model in which the spherical particle undergoing ASR, along with the ASR gel layer surrounding it, is imagined to be embedded in an infinite medium having the average properties of the composite. This approach, pursued by Xi et al. [18], is suitable for rather dilute concentrations of the particles.

In this study, we consider relatively high concentrations, for which a better idealization seems to be a periodically repetitive cubic cell of concrete of side $s$ containing only one reactive particle of initial diameter $D$ (Fig. 1); obviously $\varrho_{\mathrm{s}} \xi_{\mathrm{s}} s^{3}=\pi D^{3} / 6$ where $\varrho_{\mathrm{s}}=$ mass density of the reactive silica and $\xi_{\mathrm{s}}=$ silica concentration $=$ mass of reactive silica per unit volume of concrete $\left(\mathrm{kg} / \mathrm{m}^{3}\right)$. It follows that

$s^{3}=\frac{\pi D^{3} \varrho_{\mathrm{s}}}{6 \xi_{\mathrm{s}}}$.

A simple characterization of the diffusion of water across the growing gel layer towards the reaction front at the surface of an unreacted spherical remnant of the particle is needed. The diffusion may be assumed to be governed by a linear Fick's law. Thus, the radial flux of water $J_{w}=$ $-a_{\mathrm{s}} \operatorname{grad} \xi_{w}$ where $\xi_{w}=$ water concentration within the layer of ASR gel as a function of radial coordinate $x$, and $a_{\mathrm{s}}=$ permeability of ASR gel to water. Mass conservation within infinitesimal elements of the gel requires that $\dot{\xi}_{w}=$ $-\operatorname{div} J_{w}$ (the superior dot denotes the derivative with

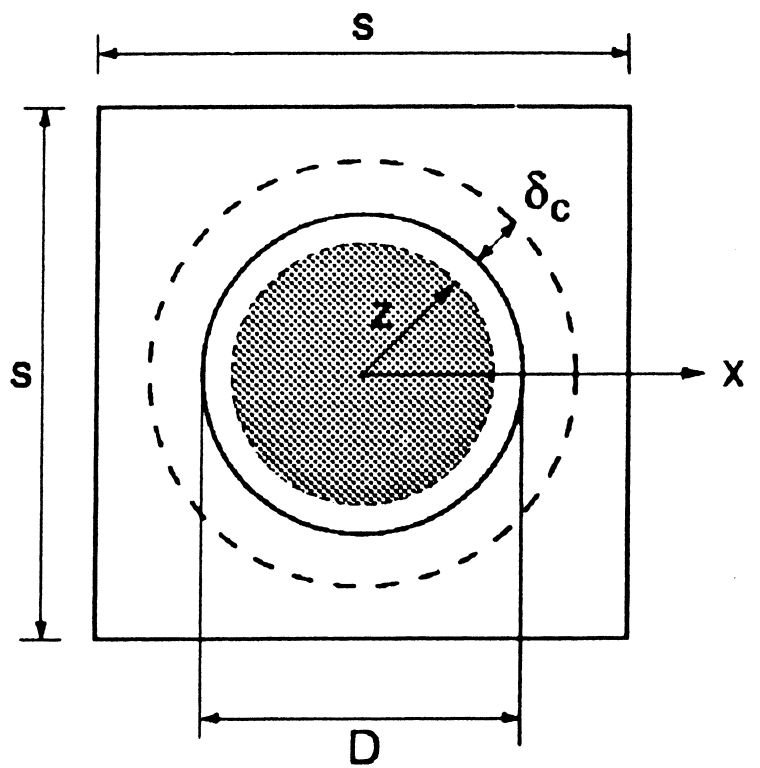

Fig. 1. Idealized repetitive cubical cell with one reactive particle of original diameter $D$ (the remaining unreacted particle of radius $z$ is marked gray; $\delta_{\mathrm{c}}$ $=$ thickness of layer with capillary pores and cracks into which pressurized ASR gel formed intrudes by diffusion. respect to time $t$ ). Therefore,

$\dot{\xi}_{w}=a_{\mathrm{s}} \operatorname{div} \operatorname{grad} \xi_{w}=a_{\mathrm{s}} \nabla^{2} \xi_{w}$.

The water diffusion and the silica dissolution at the reaction front are two coupled but distinct rate-dependent processes. The reaction at the silica dissolution front may be considered to be almost immediate compared to the duration of water transport to the front. This is justified by Dron and Brivot's ([7], p. 9, item e) observation that "the liquid film in contact with the grains of silica is at all times saturated in silica ions."

There is much glass that must undergo ASR, and so much water needs to be supplied to the reaction front. Therefore, the rate of advance of the reaction front must depend solely on the rate at which the diffusion through the ASR gel layer can supply water to the reaction front. The front can advance from an element $\mathrm{d} x$ to the next only after enough water has been supplied to combine all the silica within this element (this aspect of the problem is similar to the diffusion of pressurized water into a self-dessicated concrete of a dam [26]).

Consequently, the radial profile of $\xi_{w}$ may be expected to be almost the same as the steady-state diffusion profile $\xi_{w}$. This profile is the solution of the steady-state diffusion equation $x^{-2} \partial\left(x^{2} \partial \xi_{w} / \partial x\right) / \partial x=0$. For the proper boundary conditions, the solution yields the profile:

$\xi_{w}=w_{\mathrm{S}} F(\bar{x}), \quad F(\bar{x})=\frac{1-(2 z / D \bar{x})}{1-(2 z / D)}, \quad \bar{x}=\frac{2 x}{D}$

where $w_{\mathrm{S}}=$ concentration of water in the concrete surrounding the particle, $F(x)=$ dimensionless concentration profile, and $\bar{x}=$ dimensionless radial coordinate. For $(D / 2)-\mathrm{z}<<\mathrm{z}$ this profile approaches a linear profile, as it must.

The condition of mass balance at the reaction front $x=z$ requires that $r \varrho_{\mathrm{s}} \mathrm{d} z=-a_{\mathrm{s}}\left[\partial \xi_{w} / \partial x\right] \mathrm{d} t$ where $r=$ stoichiometric ratio (mass conversion ratio). Substituting $\xi_{w}=$ $w_{\mathrm{S}} F(\bar{x})$ according to Eq. (6) and differentiating with respect to $x$, one obtains the following differential equation [Eq. (7)] for the velocity of the reaction front:

$\dot{z}=-\frac{2 w_{\mathrm{S}} D F^{\prime}(\bar{x})}{r \rho_{\mathrm{s}} \tau_{w}}$

in which [Eq. $(8,9)]$

$$
\begin{aligned}
F^{\prime}(\bar{x}) & =\frac{\mathrm{d} F(\bar{x})}{\mathrm{d} \bar{x}} \\
& =\frac{1}{[(D / 2 z)-1] \bar{x}^{2}} \quad(z / D \leq \bar{x} \leq 1)
\end{aligned}
$$

$\tau_{\mathrm{w}}=D^{2} / a_{\mathrm{s}}$.

$\tau_{w}$ represents the halftime of diffusion of water through the gel layer, and $a_{\mathrm{s}}$ is Fick's permeability $a_{\mathrm{s}}$ (having the dimension of $\mathrm{s} / \mathrm{m}^{2}$ ). 
Note that the slope of the profile at the reaction front becomes vertical when $z \rightarrow 0$, i.e., $\lim \left[f^{\prime}(x)\right]_{x=z}=\infty$. This must be taken into account in the numerical solution.

The molar weights of the chemical species involved are

$\mathrm{SiO}_{2} \quad m_{\mathrm{s}}=60.09[\mathrm{~g} / \mathrm{mole}]$

$\mathrm{H}_{2} \mathrm{O} \quad m_{w}=18.00[\mathrm{~g} / \mathrm{mole}]$

$\mathrm{H}_{2} \mathrm{SiO}_{4}^{2-} \quad m_{\mathrm{g}}=94.10[\mathrm{~g} / \mathrm{mole}]$.

The number of moles of a certain species per $\mathrm{m}^{3}$ of concrete is $\xi / m$. According to the stoichiometric ratio of the dissolution process given by Eq. (3), the relation between the amount of water and silica involved in the reaction is [Eq. (10)]

$r=\frac{\xi_{w}}{\xi_{\mathrm{s}}}=2 \frac{m_{w}}{m_{\mathrm{s}}}$.

The mass of basic gel produced per $\mathrm{m}^{3}$ of concrete is determined by the volume of the glass that has reacted [Eq. (11)];

$\xi_{\mathrm{g}}=\frac{4 \pi}{3} \mid\left(\frac{D^{3}}{8}-z^{3}\right) \varrho_{\mathrm{s}} \frac{m_{\mathrm{g}}}{m_{\mathrm{s}}} \frac{1}{s^{3}} \quad(0 \leq z \leq D / 2)$.

\section{Modification for the case of linear flow}

The foregoing analysis may be readily adapted to the case of mineral aggregates with reactive silica uniformly dispersed in them.

In the case of mineral aggregates with veins of reactive silica, a more substantial modification is needed. In that case, the diffusion flow is better approximated as a linear (non-axisymmetric) flow across a planar layer of a certain thickness $D$. In this case, the same results are obtained except that Eq. (8) must be replaced by Eq. (12)

$F^{\prime}(\bar{x})=\frac{1}{1-2 z / D} \quad(z / D \leq \bar{x} \leq 1)$.

The gel mass produced is then given by Eq. (13)

$\xi_{\mathrm{g}}=\frac{2 z}{D} \varrho_{\mathrm{s}} \frac{m_{\mathrm{g}}}{m_{\mathrm{s}}} \quad(0 \leq z \leq D / 2)$.

\section{Swelling of ASR gel formed}

The ASR product is believed to be a solid in the absence of water; see Powers and Steinour [24]. This solid, which is here called the basic gel, has the capacity to imbibe water molecules, which is what causes the extensive swelling of the gel and makes the gel a fluid. The swelling is the typical property of colloidal systems such as the reaction product gel, and is explained by the electrical double-layer repulsion known from surface chemistry; Prezzi et al. [17].
Since the ASR gel is constrained in the pores of concrete, the swelling must produce pressure in the gel. This pressure, in turn, limits the intake of water by the gel. In fact, there must exist an equation of state giving the equilibrium specific water content of the gel as a function of water pressure and temperature, but unfortunately, this equation has apparently not yet been experimentally determined.

Expansion of the ASR gel can be partly accommodated by its expulsion into the capillary pores in the hardened cement paste located very near the surface of the particle. This is aided by the existence of an interstitial layer with a higher porosity, which is known to exist in the hardened cement paste near the aggregate surface.

As the pressure builds up, the gel migrates into these pores. The thickness, $\delta_{c}$, of the layer in which the capillary pores are accessible to the swelling ASR gel may be considered constant, independent of particle size $D$. However, the combined volume of this layer and the particle cannot exceed the volume of the cell, which gives the limitation [Eq. (14)]:

$\delta_{\mathrm{c}} \leq \frac{1}{2}(\hat{s}-D)$

where $\hat{s}=$ diameter of a sphere having the same volume as the cell; $\pi \hat{s}^{3} / 6=s^{3}$ or

$\hat{s}=s(6 / \pi)^{1 / 3}$.

As already mentioned, the physical properties of the basic form of ASR gel are not yet known. But it seems logical to assume that the formation of this basic gel per se causes no significant volume increase. So, it appears reasonable to assume that any swelling of the ASR gel is caused solely by the intake of additional water.

Denoting by $w_{\mathrm{i}}$ [Eq. (16)] the mass of water imbibed by the basic gel, the volume increase of the gel is

$\Delta V=\frac{w_{\mathrm{i}}}{\varrho_{w}}$.

Since the gel is constrained in the concrete, this increase in volume produces pressure in the gel which depends on the gel compressibility $\kappa_{\mathrm{g}}$ [Eq. (17)]

$p=\kappa_{\mathrm{g}} \Delta V$.

The imbibition of water from the bulk of mortar or concrete in the cell into the basic gel cannot happen instantly. It is a local micro-diffusion process that occurs with some delay. Because the gel is expelled into the pores, the geometry of this diffusion is no longer spherical and it appears difficult to choose any particular idealized geometry of the diffusion flow of water that should be analyzed. It seems therefore appropriate to conduct merely a simplified overall analysis without reference to any particular geometry. It is reasonable to assume that in Eq. (18)

$\dot{w}_{\mathrm{i}}=A_{\mathrm{i}} / \tau_{\mathrm{i}}$ 
where $A_{\mathrm{i}}$ characterizes the thermodynamic affinity (difference in chemical potentials, per unit surface area of particle) in terms of a concentration difference, and $\tau_{i}$ is a certain time constant.

To set up a reasonable expression for $A_{\mathrm{i}}$, several facts should now be noted.

(1) The chemical potential (Gibbs' free energy per unit mass) governing water transport through concrete may be assumed to be roughly proportional to the specific water content $w_{\mathrm{i}}$, or else Fick's law of diffusion could not be valid.

(2) The water imbibition rate $\dot{w}_{\mathrm{i}}$, and thus $A_{\mathrm{i}}$, should be proportional to the mass of basic gel (per cell), $\xi_{\mathrm{g}}$.

(3) $\dot{w}_{\mathrm{i}}$ must decrease with increasing pressure $p$ in the gel, the pressure being caused by the elastic resistance of concrete to swelling, which may be described by some empirical decreasing function $n(p)$ (Fig. 2a). Thus, function $n$ gives the specific mass of water that is imbibed by the basic gel at a certain pressure when thermodynamic equilibrium is reached.

(4) $\dot{w}_{\mathrm{i}}$ must decrease with a decreasing relative humidity $h$ in the pores of concrete near the reacting particle. The imbibition must stop at a certain pore humidity such as $h$ $=0.85$ representing a swelling threshold (Fig. 2b). The existence of such a threshold is indicated by the fact that no swelling due to ASR is observed in concrete exposed to an environment of relative humidity less than about $85 \%$.

According to these facts, the chemical potential of the water imbibed by the gel may be considered approximately proportional to $n(p) m(h) \xi_{\mathrm{g}}$. This represents the value of $w_{\mathrm{i}}$ at thermodynamic equilibrium, at which no water migrates into or out of the ASR gel. Therefore, the affinity (thermodynamic driving force or chemical potential difference) governing the water imbibition may be approximately expressed as Eq. (19)

$A_{\mathrm{i}}=n(p) m(h) \xi_{\mathrm{g}}-w_{\mathrm{i}}$.

For reasons of dimensionality, the time constant, called the imbibition halftime, must have the basic form $\tau_{\mathrm{i}}=\delta^{2} / C_{w_{\mathrm{i}}}$

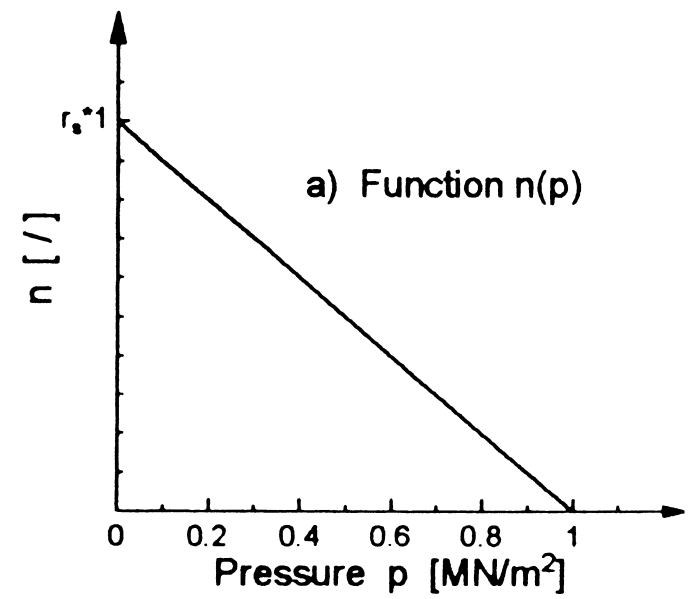

where $C_{w_{i}}$ is the diffusivity for microdiffusion of water within the cell (surely much lower than the diffusivity for global water diffusion through a concrete structure), and $\delta$ is the average (or effective) distance of water transport from the concrete in the cell into the ASR gel. Roughly, $\delta=\hat{s}-D) / 2$ where $\hat{s}=s(6 / \pi)^{1 / 3}$ of Eq. (15) represents the diameter of a sphere having the same volume as the cell, $s^{3}$. However, if the layer $\delta_{\mathrm{c}}$ protrudes into the adjacent cell, the protruding part may not be counted, therefore,

$\tau_{\mathrm{i}}=\frac{1}{C_{w_{\mathrm{i}}}} \mid\left\{\min \mid\left(\frac{\hat{s}-D}{2}, \delta_{\mathrm{c}}\right)\right\}^{2}$.

The fact that the total water $w_{\mathrm{i}}$ in the cell will govern the imbibition rate only after a roughly uniform gradient of the driving force (chemical potential) develops is ignored in Eq. (20), as a simplification.

Putting all these effects together, one has the following approximate rate equation $[\mathrm{Eq}(21)]$ for water imbibition into the gel:

$$
\left.\dot{w}_{\mathrm{i}}=\underset{\times\left[n(p) m(h) \xi_{\mathrm{g}}-w_{\mathrm{i}}\right] .}{\mid} \min \left|\left\{1, \mid\left(\frac{D+2 \delta_{\mathrm{c}}}{\hat{s}}\right)^{3}\right\}-\right|\left(\frac{D}{\hat{s}}\right)^{3}\right] \frac{1}{\tau_{\mathrm{i}}}
$$

Experimental observations suggest that even the formation of the basic (unswolen) ASR gel is arrested when the relative humidity $h$ in the capillary pores drops below about $85 \%$. The reason must be that the chemical potential of water at the surface of ASR gel is proportional not to the overall specific water content $w$ of concrete but to $m(h) w$. Therefore, it seems reasonable to introduce the boundary condition:

$w_{\mathrm{S}}=m(h) w$.

This boundary condition [Eq. (22)] will cause that not only the water imbibition into the existing ASR gel but also the

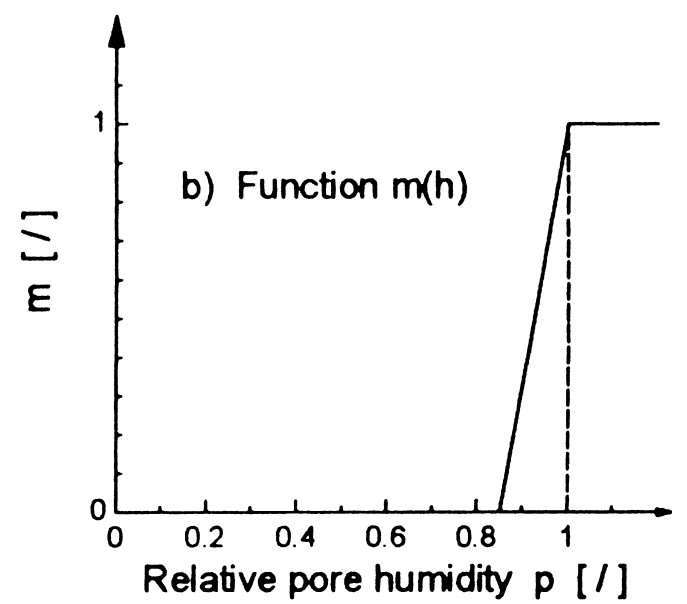

Fig. 2. (a) Function $n(p)$ describing the influence of gel pressure equilibrium content of imbibed water; (b) function $m(h)$ describing the influence of relative pore humidity on the imbibition of water by the gel. 
formation of further basic ASR gel will stop when the relative humidity in the capillary pores drops below $85 \%$.

The explanation of Eq. (22) is that, at low humidity $h$, much of the remaining pore water is strongly held in the form of adsorbed water in the micropores of cement gel and the remaining capillary water is strongly held in very narrow pores with capillary meniscus of a very small radius and very high surface tension. Consequently, the pore water present is unavailable for the reaction. It cannot migrate to the surface of the ASR gel.

It might be questioned why two separate diffusions of water are considered. The reason is that only the second diffusion leading to water imbibition of the ASR gel represents a diffusion of water per se. The diffusion of water through the basic gel toward the remnant of glass particle is nothing but a simplified substitute for the diffusion of ions toward the reaction front. Formulating the diffusion of ions mathematically would of course be more realistic, but also more complicated. Because a movement of ions represents an electric current, it would then be necessary to analyze these currents and solve the electric potential field.

\section{Water supply by macroscopic diffusion}

With the foregoing equations, the main phenomena and reactions are described on a microscopic scale considering one reactive particle in a unit cell. Since the process of ASR depends strongly on the supply of water, the macroscopic diffusion of water through the concrete also needs to be modeled. Denoted by $h$, the relative vapor pressure (humidity) in the capillary pores of concrete, the macrodiffusion of water through the concrete can be described by a linear Darcy's law [Eq. (23)]

$J_{w}=-a(h) \operatorname{grad} h$

where $a(h)$, the permeability of concrete, which decreases about 20 times as $h$ drops from $100 \%$ to $60 \%$ [27-30]. Taking into account the mass conservation condition yields the following non-linear diffusion equation [Eq. (24)]:

$\dot{h}=-k(h) \operatorname{div} J_{w}-\dot{h}_{\mathrm{s}}\left(t_{\mathrm{e}}\right)-k(h)\left(\dot{w}_{\mathrm{g}}+\dot{w}_{\mathrm{i}}\right)$.

The self-desiccation of the concrete, which depends on the equivalent hydration time $t_{\mathrm{e}}$ (equivalent curing period) is here taken into account by the sink term $h_{\mathrm{s}}\left(t_{\mathrm{e}}\right)$ representing a given function of time. The formation of the basic gel consumes water that is withdrawn from the capillary pores, and so it represents a sink term, $w_{\mathrm{g}}$. The additional water that is imbibed by the ASR gel is also withdrawn from the capillary pores and so it represents a third sink term $w_{\mathrm{i}}$. Function $k(h)$ represents the inverse slope of the desorption isotherm of concrete; it represents the ratio of the pore humidity increment $\mathrm{d} h$ to the water content increment $\mathrm{d} w$ (per unit volume of concrete) in thermodynamic equilibrium.
The values of the parameters of this diffusion model are given in Refs. [27,30] A more refined mathematical model for water diffusion in concrete was presented in Refs. $[28,29]$.

\section{Parametric study of numerical solutions}

A numerical step-by-step integration of the system of differential equations presented here has been programmed. The increments of $\xi_{w}$ have been calculated from Eq. (5) as the difference between the values of $\xi_{w}$ at two subsequent discrete times. The finite difference method has been used for spatial approximations.

Examples of practical predictions would not be meaningful at this time because experimental information on various vital parameters of the present model is lacking. Therefore, only the effects of various parameters have been studied. The results are plotted in Figs. 3-5. The following observations can be made.

(1) The build-up of pressure due to the swelling of the gel depends strongly on the size $D$ of the reactive particle (Fig. 3a). For larger particles, the pressure increases as the particle size decreases. However, there exists a certain pessimum size for which the pressure is maximum. Reducing the particle size further causes a decrease of pressure.

(2) In agreement with the topochemical aspect of the reaction producing the basic gel, it is found that the gel formation accelerates with an increasing overall surface of the reactive particles, i.e., with a decreasing particle size at constant volume fraction of the reactive silica in concrete (Fig. 3b).

(3) As expected from the fact that the formation of basic gel consumes water, the relative pore humidity is lower when the particles are smaller (Fig. 3c). Thus, if pore humidity drops below the threshold of $85 \%$, no water is imbibed by the basic gel and, accordingly, no increase in pressure is observed. This explains why, when focusing for example on particle diameter $D=0.001 \mathrm{~m}$ in Fig. 3a, the pressure should be less for a higher amount of reactive aggregate.

(4) Fig. 3a further shows that the pessimum value of particle size also depends on the mass concentration $\xi_{\mathrm{s}}$ of the reactive silica.

(5) An increase of the permeability of basic gel accelerates the formation of basic gel (Fig. 4a), and thus causes a drop in pore humidity below the threshold for swelling. The pessimum value of particle diameter increases with increasing permeability (Fig. 4b).

(6) The permeability of concrete has an important influence on the progress of ASR. This is shown by an example of a 1-m thick wall under ASR attack (the wall surface is at $x=0$, the plane of symmetry at $x=0.5$; thanks to symmetry, only the half thickness $0.5 \mathrm{~m}$ is analyzed numerically). Fig. 5 a shows the influence of concrete permeability $a(h)$ on the 

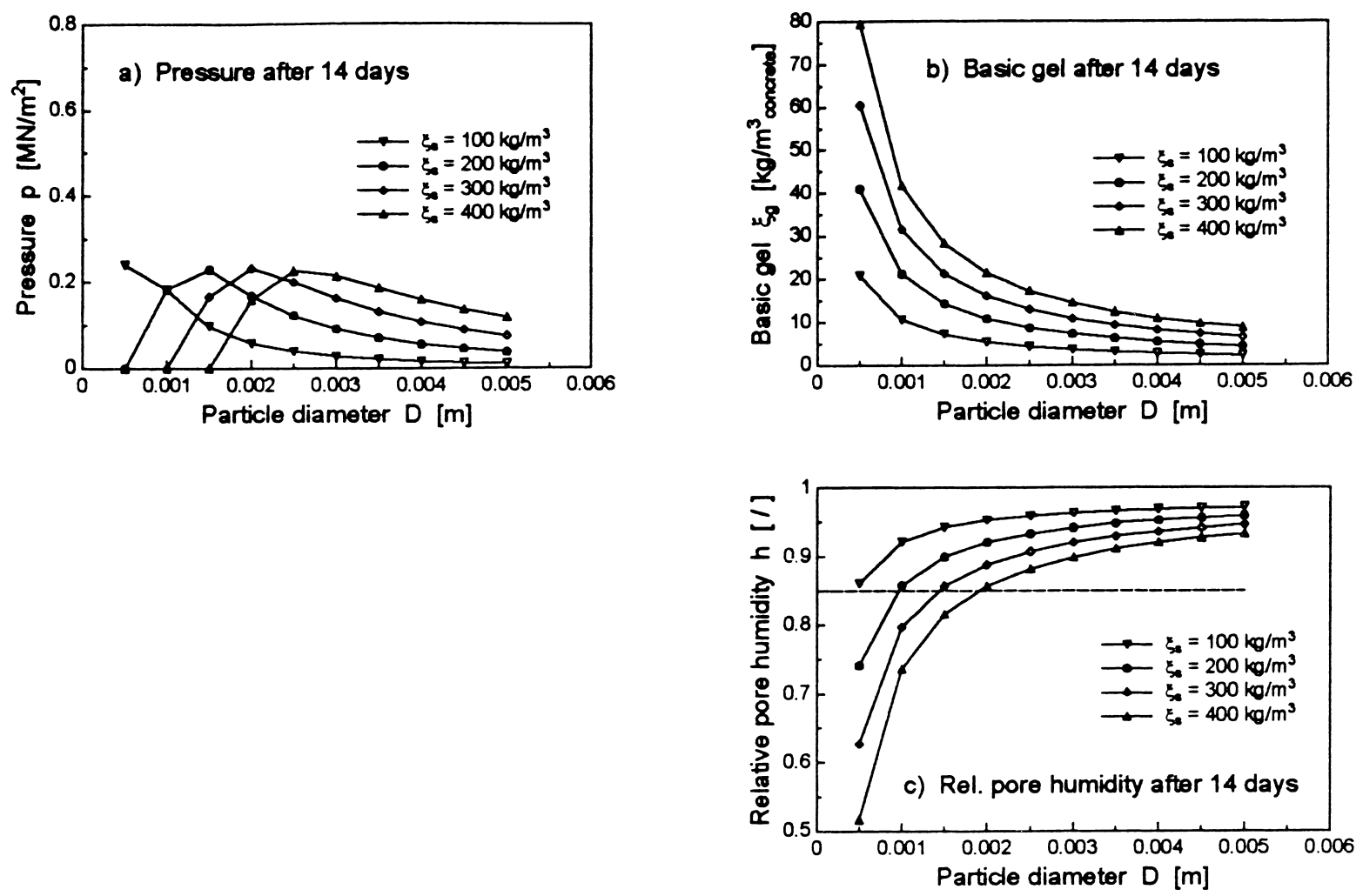

Fig. 3. Influence of reactive particle diameter $D$, at various concentrations $\xi_{\mathrm{s}}$ of reactive silica, on: (a) pressure $p$, (b) relative pore humidity $h$, and (c) the mass of basic gel $\xi_{\mathrm{g}}$ formed by ASR.

pressure distribution in the wall at two different diameters $D$ of reactive particles. When the permeability is higher, the zone of swelling of the gel reaches deeper into the wall. This is caused by the fact that the water necessary for the reaction is supplied faster.

(7) The particles of diameter $D=0.002 \mathrm{~m}$ cause a high pressure throughout the whole wall, whereas the smaller particles with $D=0.001 \mathrm{~m}$ caused a high gel pressure $p$ only in the zone near the surface. The plot of the relative pore humidity in the specimen (Fig. 5b) shows the drop in pore humidity for small particles, which in turn limits the swel- ling of the basic gel. This explains why the build-up of pressure is restricted to the surface zone if the reactive particles are small enough. It also shows a faster supply of water to deeper zones in the structure.

\section{Relation to some previous studies}

The existence of a certain pessimum reactive particle size below which a decrease of particle size reduces the deleterious effects of ASR was experimentally discovered by
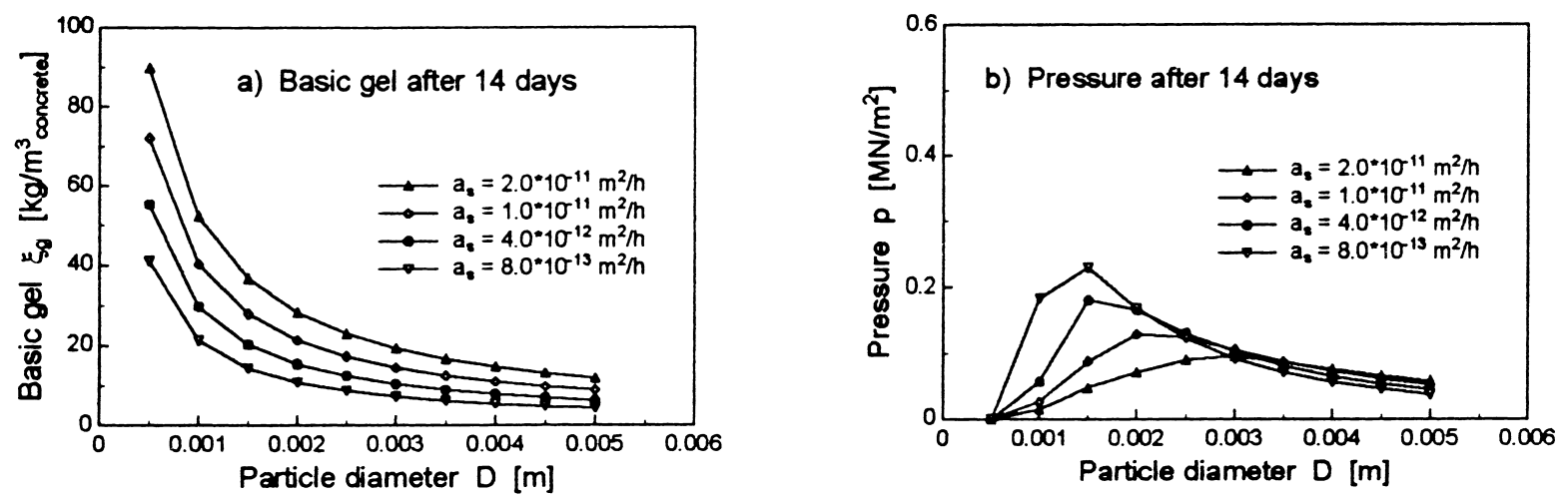

Fig. 4. Influence of particle diameter $D$, at various values of permeability $a_{\mathrm{s}}$ of the basic gel, on (a) mass $\xi_{\mathrm{g}}$ of basic gel formed by ASR, and (b) pressure $p$. 

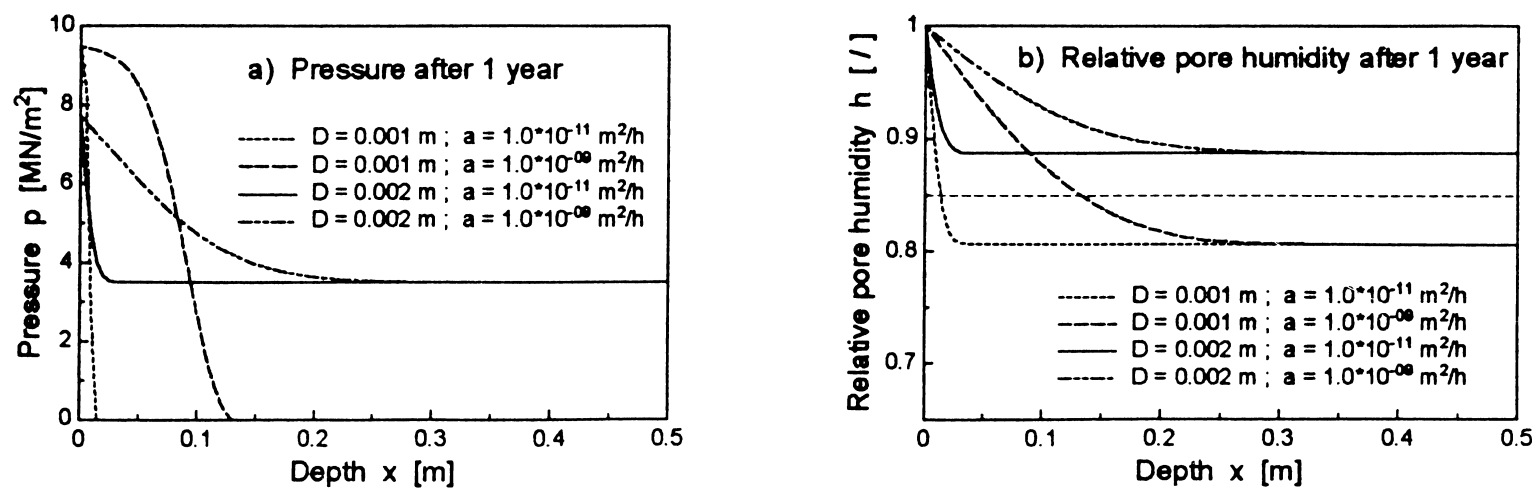

Fig. 5. Influence of reactive particle diameter $D$ and bulk concrete permeability $a(h)$ on the ASR attack on a wall of 1-m thickness (thanks to symmetry, only $0.5 \mathrm{~m}$ is shown).

Meyer and Baxter [22] and Jin [23] at Columbia University. As seen in the parametric study just described, the present model of ASR kinetics can explain this initially startling discovery, which suggests the waste glass could be incorporated in concrete if it is ground to sufficiently small particles. The explanation of this discovery based on diffusion analysis (albeit more simplified than the present model) was previously also suggested by Xi et al. [18].

ASR kinetics, however, is not the only explanation. The existence of the pessimum particle size can also be explained by fracture mechanics, as shown in Ref. [21]. Obviously, a combination of the fracture mechanics approach with the present ASR kinetics approach could be expected to provide a similar dependence of gel pressure and concrete expansion on the particle size, and in particular, to retain the existence of pessimum size in the combined model. The development of a combined model is the objective of a proposed project.

After the present calculations had been completed, Xi et al. [18] published valuable modeling studies that exhibit some similarities as well as significant differences. Aside from idealizing the situation by the self-consistent model for infinite medium instead of a periodically repetitive cell, $\mathrm{Xi}$ et al. [18] refined a preceding model by Groves and Zhang [16] and considered separate diffusions of alkali ions and hydroxyl ions toward the reaction front advancing radially into in a reactive sphere. They do not consider the dependence of the rate of ASR gel formation on the pore relative humidity [function $n(h)$ ], nor the macroscopic diffusion through concrete that supplies water to the reaction. Their highly interesting study is focused on the use of fly ash to mitigate the volume changes in the presence of ASR.

\section{Summary and conclusions}

The main motivation of the present model is the goal of using waste glass as a substitution for a part of the aggregate in concrete, although in general, the model can also be applied to reactive mineral aggregates. A relatively simple model for the kinetics of ASR in concrete is developed by analyzing an idealized repetitive cubical cell with one spherical reactive particle.

In the case of waste glass, the particle consists entirely of reactive silica. The radial growth of a spherical layer of basic ASR gel into the reactive particle may be assumed to be controlled by the diffusion of water through the gel layer toward the reaction front. The swelling of the gel layer is due to the imbibition of water from the adjacent capillary pores, which represents another diffusion process.

The swelling is resisted by concrete which causes development of pressure. The pressure build-up is considered to inhibit water imbibition and to cause part of the gel to diffuse into the adjacent capillary pores and cracks in the concrete matrix. The diffusivities are assumed to depend on the relative humidity in the capillary pores. The supply of water to the cell is calculated from the macroscopic non-linear diffusion equation for the transport of water through concrete.

Parametric study of the numerical solutions of the system of differential equations of the problem clarifies the effects of particle size. In particular, it shows that there exists a certain pessimum size of the reactive particle below which the effect of particle size is reversed-the maximum pressure reached decreases rather than increases with the particle size. This finding agrees with the previous experimental results of C. Meyer et al. at Columbia University. The existence of pessimum particle size is also predicted by a previous fracture mechanics approach, which is planned to be combined with the present model in future research.

The effects of mass concentration of reactive silica in concrete and of the permeabilities of ASR gel and of bulk concrete, as well as the dependence of ASR on the pore humidity evolution, are also studied.

\section{Acknowledgments}

Thanks are due to the US National Science Foundation for partial support under Grant CMS-9713944 to North- 
western University. Thanks are due also to Franz-Josef Ulm, MIT, for very valuable discussions.

\section{References}

[1] T.H. Stanton, Expansion of concrete through reaction between cement and aggregate, Proc Am Soc Civil Eng 66 (1940) 1781-1811.

[2] R.F. Blanks, Concrete deterioration at Parker Dam, Eng News-Rec 126 (1941) $462-465$.

[3] H.S. Meissner, Cracking in concrete due to expansion reaction between aggregate and high-alkali cement as evidenced in Parker Dam, Proc ACI 37 (1941) 549-568.

[4] F.P. Glasser, Chemistry of the alkali aggregate reaction, in: R.N. Swamy (Ed.), The Alkali-Silica Reaction in Concrete, Blackie, Glasgow and London, and Van Nostrand-Reinhold, New York, 1992, pp. 30-53.

[5] L.S. Dent Glasser, N. Kataoka, Alkali-Aggregate-Reaction in Concrete, Paper S 252/23, CSIR, Pretoria, 1981.

[6] R. Dron, F. Brivot, Thermodynamic and kinetic approach to the alkali-silica reaction: Part 1, Cem Concr Res 22 (5) (1992) 941-948.

[7] R. Dron, F. Brivot, Thermodynamic and kinetic approach to the alkali-silica reaction: Part 2, Cem Concr Res 23 (4) (1993) 1001-1002.

[8] R. Dron, F. Brivot, Solid-liquid equilibria in K-C-S- $\mathrm{H} / \mathrm{H}_{2} \mathrm{O}$ systems, Proc., 10th Intern. Conf. on Alkali-Aggregate Reaction in Concrete, Melbourne.

[9] R. Dron, F. Brivot, T. Chaussadent, Mechanism of the alkali-silica reaction, 10th Int. Conf. on the Chem. of Cem., Gothenburg, Sweden, Vol. 4.

[10] H.E. Vivian, Studies in cement aggregate reaction: XVI. The effect of hydroxyl ions on the reaction of opal, Aust J Appl Sci 2 (1951) $108-113$.

[11] E.G. Swenson, A reactive aggregate undetected by ASTM tests, ASTM Bull 226 (1957) 48-57.

[12] A.B. Poole, Introduction to alkali-aggregrate reaction in concrete, in: R.N. Swamy (Ed.), The Alkali-Silica Reaction in Concrete, Blackie, Glasgow and London, and Van Nostrand-Reinhold, New York, 1992, pp. $1-29$.

[13] R.N. Swamy, Testing for alkali-silica reaction, in: R.N. Swamy (Ed.), The Alkali-Silica Reaction in Concrete, Blackie, Glasgow and London, and Van Nostrand-Reinhold, New York, 1992, pp. 54-95.

[14] R.N. Swamy (Ed.), The Alkali-Silica Reaction in Concrete, Blackie, Glasgow and London, and Van Nostrand-Reinhold, New York, 1992.

[15] E.M. Gartner, H.M. Jennings, Thermodynamics of calcium-silicate hydrates and their solutions, J Am Ceram Soc 70 (10) (1987) $743-749$.

[16] G.W. Groves, X. Zhang, A dilatation model for the expansion of silica glass/OPC mortars, Cem Concr Res 20 (1990) 453-460.
[17] M. Prezzi, P.J.M. Monteiro, G. Sposito, The alkali-silica reaction: Part I. Use of double-layer theory to explain the behavior of reaction product gels, ACI Mater J 123 (1997) 10-11.

[18] Y. Xi, A. Suwito, X. Wen, C. Meyer, W. Jin, Testing and modeling of alkali-silica reaction and the associated expansion of concrete, in: G. Pijaudier-Cabot, Z. Bittnar, B. Gérard (Eds.), Mechanics of QuasiBrittle Materials and Structures: A Volume in Honor of Prof. Z.P. Bažant's 60th Birthday, Hermes Science Publications, Paris, 1999, pp. 217-232.

[19] Z.P. Bažant, W. Jin, C. Meyer, Micromechanical fracture analysis of ASR-induced damage in concrete with waste glass, in: H. Murakami, J.E. Luco (Eds.), Engineering Mechanics: A Force for the 21st Century, Proc. 12th Eng. Mech. Conf., held in La Jolla, CA. ASCE, Reston, VA, 1998a, pp. 48-51.

[20] Z.P. Bažant, W. Jin, C. Meyer, Microfracturing caused by alkali-silica reaction of waste glass in concrete, 3rd Int. Conf., FraMCoS-3, Gifu, Japan.H. Mihashi, K. Rokugo (Eds.), Fracture Mechanics of Concrete Structures, Vol. 3, Aedificatio Publishers, Freiburg, Germany, 1998b, pp. $1687-1694$.

[21] Z.P. Bažant, G. Zi, C. Meyer, Fracture mechanics of alkali-silica reaction in concretes with waste glass particles of different sizes, ASCE J Eng Mech, 126 (3) (2000) 226-232.

[22] C. Meyer, S. Baxter, Use of recycled glass and fly ash for precast concrete. Report NYSERDA 98-18 (4292-IABR-IA-96) to New York State Energy Research and Development Authority, Dept. of Civil Eng. and Eng. Mech., Columbia University, 1998.

[23] W.-H. Jin, Alkali-silica reaction in concrete with glass aggregate-a chemo-physical-mechanical approach. PhD Dissertation, Columbia University, New York, 1998.

[24] T.C. Powers, H.H. Steinour, J. Am. Concr. Inst. (Proc.) 55 (1955) $497-516$ and $785-812$.

[25] L.S. Dent Glasser, N. Kataoka, On the role of Calcium in the Alkaliaggregate reaction, Chem Concr Res 12 (1982) 321.

[26] Z.P. Bažant, Pore Pressure, Uplift, and Failure Analysis of Dams, Proc., Symp. on Criteria and Assumptions for Numerical Analysis of Dams (held at Univ. of Wales, Swansea). Brit. Nat. Committee on Large Dams, 1975, pp. 781-808.

[27] Z.P. Bažant, L.J. Najjar, Drying of concrete as a nonlinear diffusion problem, Cem Concr Res 1 (1971) 461-473.

[28] Y. Xi, Z.P. Bažant, H.M. Jennings, Moisture diffusion in cementitious materials: Absorption isotherms, Adv Cem Based Mater 1 (1994a) $248-257$

[29] Y. Xi, Z.P. Bažant, L. Molina, H.M. Jennings, Moisture diffusion in cementitious materials: Moisture capacity and diffusivity, Adv Cem Based Mater 1 (1994b) 258-266.

[30] Z.P. Bažant, M.F. Kaplan, Concrete at High Temperatures: Material Properties and Mathematical Models, Longman (Addison-Wesley), London, 1996. 